\title{
Neural correlates of evaluation associated with promotion and prevention regulatory focus
}

\author{
WILLIAM A. CUNNINGHAM \\ University of Toronto, Toronto, Ontario, Canada \\ and \\ CAROL L. RAYE and MARCIA K. JOHNSON \\ Yale University, New Haven, Connecticut
}

\begin{abstract}
Higgins (1997, 1998) proposed two self-regulatory or motivational systems-one sensitive to gains (promotion) and one sensitive to losses (prevention). To examine the interaction of motivation and cognition, participants made good/bad or abstract/concrete judgments about concepts during fMRI scanning. After scanning, participants rated the extent to which each stimulus was good and bad and completed a questionnaire that measured promotion/prevention orientation. For each participant, contrast maps were generated representing the association between neural processing and stimulus valence (good/bad), and these factors were then regressed against participants' promotion and prevention focus scores. For the good/bad but not for the abstract/concrete task, promotion focus was associated with greater activity in the amygdala, anterior cingulate, and extrastriate cortex for positive stimuli, and prevention focus was associated with activity in the same regions for negative stimuli; these results are consistent with the hypothesis that the way in which evaluative information is processed is influenced by individual differences in self-regulatory focus.
\end{abstract}

Evaluative processes allow people to make choices that will maximize gains and minimize costs. Such evaluations can focus on the attainment of positive outcomes and/or the avoidance of negative outcomes. That is, people can make decisions on the basis of either gain/nongain or loss/nonloss dimensions and attain the same relative utility. Moreover, if people habitually vary in their weighting of the ratio of positivity and negativity, it would lead to quite different styles of evaluative judgment. That is, some people may be relatively more motivated by negative stimuli or events and others more by positive stimuli or events.

Higgins $(1997,1998)$ proposed that there are two selfregulatory or motivational systems - one sensitive to gains (promotion) and one sensitive to losses (prevention). A promotion orientation is associated with seeking advancement and accomplishment, whereas a prevention orientation is associated with concerns of safety and responsibility. In terms of this proposal, quite different information in the environment can be important for different judgments. For example, positive information should be more important for promotion than for prevention focus, and negative information should be more

This research was supported by a grant from the National Institutes of Health (MH 62196). We thank Jay Van Bavel, Phil Zelazo, and Norman Farb for helpful comments on an earlier version of this article. Correspondence relating to this article may be sent to W. A. Cunningham, Department of Psychology, University of Toronto, 100 St. George Street, Toronto, ON, M5S 3G3 Canada (e-mail: cunningham@psych. utoronto.ca). important for prevention than for promotion focus. In support of this idea, when participants imagined future outcomes, positive outcomes were perceived to be better by those with a promotion rather than a prevention focus, and negative outcomes were perceived to be worse by people with a prevention rather than a promotion focus (Idson, Liberman, \& Higgins, 2000). Relative to prevention focus participants, those induced to have a promotion focus enjoyed a task in which they needed to find helpful objects. The reverse pattern was found for prevention focus participants; for them, a task in which they found harmful objects was more enjoyable (Freitas \& Higgins, 2002).

In addition, promotion focus and prevention focus lead to differences in the means of goal pursuit: Promotion focus leads to eagerness, and prevention focus leads to vigilance (Higgins, 2000). When the means of goal pursuit match self-regulatory focus (e.g., when a task is framed in terms of vigilance for a prevention-focused participant), people are more motivated to engage in a task, perform it better, and place more value on the outcome than when the means do not fit the self-regulatory focus (e.g., if the task above were framed in terms of eagerness) (Higgins, Idson, Freitas, Spiegel, \& Molden, 2003; Spiegel, Grant-Pillow, \& Higgins, 2004). In signal detection terms, promotion focus would be associated with ensuring hits and avoiding errors of omission (the gain/ nongain dimension); to achieve and advance, one must find and not miss opportunities. Prevention focus, in contrast, would be associated with ensuring correct rejections and avoiding errors of commission (the loss/nonloss dimen- 
sion); to achieve security, one must be sure to identify and avoid punishments. In the attitude domain, persuasive messages in which regulatory focus is paired with an appropriate frame (for promotion, gain/nongain; for prevention, loss/nonloss) are more likely to result in attitude change (Cesario, Grant, \& Higgins, 2004; Lee \& Aaker, 2004). Together, these findings indicate that differences in promotion versus prevention focus influence behavior in a wide range of circumstances.

Although self-regulatory focus is conceptualized at the level of motivational systems, it is closely associated with aspects of approach and avoidance behavior. Gray (1981, 1982) suggested that two motivational systems underlie behavior and affect: a behavioral inhibition system (BIS) and a behavioral approach/activation system (BAS). Individual differences in BIS/BAS scores have been shown to be associated with differences in brain activity when processing valenced stimuli. For example, Reuter et al. (2004) found that BIS scores are correlated with activity in anterior cingulate, amygdala, and thalamus when looking at emotion-invoking pictures. Interestingly, when their results were collapsed across picture types (erotic, fearful, disgust), no consistent pattern emerged as being associated with individual differences in BAS scores. Although it is tempting to equate promotion focus with an approach system and prevention focus with an avoidance system, the difference between self-regulatory focus and BIS/BAS is important. Self-regulatory focus is a higher order motivational state that directs focus of attention and evaluation, which in turn direct behavior, including approach or avoidance behavior.

Other individual differences in personality have also been associated with differences in brain activity when processing valenced stimuli. For example, degree of extroversion has been shown to be associated with greater activation in the amygdala when participants view pleasant photographs (Canli et al., 2001) or happy faces (Canli, Sivers, Whitfield, Gotlib, \& Gabrieli, 2002). In addition, individual differences in neuroticism (a Big-5 personality trait) have been associated with increased activity in the left middle temporal gyrus and the left middle frontal gyrus when viewing negative images (Canli et al., 2001). The Big-5 model is a descriptive rather than a process model (Pervin, 1994), however, and little research has examined individual differences in motivation and cognition during evaluation. ${ }^{1}$ Specifically, it remains unknown how or whether different individuals' self-regulatory orientations are reflected in differences in neural activity during the evaluation of stimuli. In this study, we examined how promotion and prevention orientations are associated with differences in brain activity during the evaluation of stimuli.

Previous work has suggested that several brain regions are involved in evaluative processing in general. For example, numerous studies have indicated that the amygdala is critical for some aspects of evaluation. Across multiple perceptual modalities, greater amygdala activation has been associated with processing negative in comparison with positive stimuli (Cunningham, Johnson, Gatenby, Gore, \& Banaji, 2003; Isenberg et al., 1999; LaBar, Gatenby, Gore, LeDoux, \& Phelps, 1998; LeDoux, 2000; Morris et al., 1996; Phelps et al., 2001; Zald, Lee, Fluegel, \& Pardo, 1998; Zald \& Pardo, 1997). Moreover, patients with amygdala damage show impairments in fear conditioning and perception of emotional stimuli (Adolphs \& Tranel, 2004; Adolphs et al., 1999; Anderson, Spencer, Fulbright, \& Phelps, 2000; LaBar, LeDoux, Spencer, \& Phelps, 1995) and impaired perceptual attention to arousing stimuli (Anderson \& Phelps, 2001).

However, studies have also found greater amygdala activation for positive as compared with neutral stimuli (Garavan, Pendergrass, Ross, Stein, \& Risinger, 2001; Hamann, Ely, Hoffman, \& Kilts, 2002; Hamann \& Mao, 2002; Liberzon, Phan, Decker, \& Taylor, 2003), and when overall emotional intensity is controlled, no relationship remains between negativity/positivity and amygdala activation (Cunningham, Raye, \& Johnson, 2004). Cunningham et al. (2004) found that amygdala activity is sensitive to emotional intensity rather than the "badness" or "goodness" of a stimulus. This pattern of results suggests that the amygdala may be involved in the processing of important, or motivationally relevant, stimuli rather than negativity or positivity per se. Indeed, Whalen (1998; Davis \& Whalen, 2001) has suggested that the primary role of the amygdala is in increasing vigilance when necessary. The greater association of amygdala activation with negative than with positive stimuli could reflect an automatic capture of attention selected for by evolutionary or genetic forces, if negative stimuli have a relatively larger influence on survival, or alternatively it could reflect a habitual vigilance for negative stimuli developed through individual experience. If the latter theory is true, as is suggested by selfregulatory focus theory, there should be people for whom and/or times when positive stimuli take on relatively more important motivational relevance-for example, when a person has promotion rather than prevention focus. Thus, self-regulatory focus should be associated with relatively greater attention to positive stimuli for promotion focus and to negative stimuli for prevention focus.

In order to examine how individual differences in selfregulatory orientation are associated with differences in the neural processing of evaluative information, during fMRI scanning we asked participants to make good/bad and abstract/concrete judgments about concepts (e.g., babies, guns, murder, recycling, immigration). After scanning, participants rated each stimulus for goodness, badness, and emotional intensity and completed a short questionnaire that measured both promotion and prevention regulatory orientation. Using regression analysis, we examined the relationship between prevention and promotion orientation and the neural processing of valence. Positive relationships with either prevention or promotion scores indicate that a brain region is associated with the processing of positive stimuli. Negative re- 
lationships indicate that a brain region is associated with the processing of negative stimuli.

\section{METHOD}

\section{Participants}

Twenty participants ( 8 male, 12 female, mean age $=21$ years $)$ were paid for their participation. The participants reported no abnormal neurological history and had normal or corrected-to-normal vision. All participants provided informed consent.

\section{Materials}

A total of 144 concepts were selected for the study. These concepts were chosen to vary on multiple dimensions, such as $\mathrm{good} / \mathrm{bad}$, abstract/concrete, and emotionality. Examples of concepts used in the study include "babies," "cigarettes," "freedom," "garbage," "guns," "love," "murder," "poetry," "recycling," "technology," and "terrorism." A four-item questionnaire was developed for a quick assessment of self-regulatory focus: I focus on opportunities that will enhance my life; I focus on ensuring that I will avoid potential mishaps or negative events; I am primarily motivated by seeking potential successes; I am primarily motivated by avoiding failure. The participants answered each item on a 6-point scale anchored by strong disagree (1) and strong agree (6). A participant's promotion score was the average of the first and third items, and the participant's prevention score was the average of the second and fourth items. The scale was scored so that 6 represented the highest degree of a regulatory focus and 1 represented the lowest. Testing has demonstrated that this four-item questionnaire is highly correlated with other measures of self-regulatory focus. In fact, in a multitrait multimethod examination of the scales' validity, this measure was at least as valid, if not more so, than other available measures (Farb \& Cunningham, 2005). The mean promotion focus for this sample was $4.82(S D=0.90)$, and the mean prevention focus was $4.13(S D=0.80)$.

\section{Procedure}

On each fMRI trial, the participants categorized a concept according to one of two dimensions (good/bad or abstract/concrete) and indicated their responses by making one of two buttonpresses with the right hand. Using E-Prime software for PC (Psychology Software Tools, Inc.; Pittsburgh, PA), stimuli were forward projected with an LCD onto a screen by the participants' feet. A prism mirror positioned over the participants' eyes allowed them to view the stimuli. All stimuli were presented in black letters against a white background.

On each trial, a 500-msec cue indicated whether the trial required a good/bad or an abstract/concrete judgment, immediately followed by a concept presented for $2 \mathrm{sec}$, during which the participants made their response. Additional null fixation trials were used to space out experimental trials. The ratio of critical trials to null fixation trials was $4: 1$. Between all trials, a fixation cross remained on the screen for 2, 4, or $6 \mathrm{sec}$. From the participants' point of view, a null trial was a longer fixation between trials. To synchronize stimulus presentations with functional scanning, all functional runs were initiated by a trigger sent by the MRI scanner. Each of six runs contained 24 of each trial type (good/bad task, abstract/concrete task), randomly intermixed. All 144 concepts were presented before any were repeated. Two lists were counterbalanced across participants so that each concept was first rated as good/bad for half of the participants and as abstract/concrete for the other half.

After scanning, the participants completed a questionnaire in which all concepts were rated separately for goodness, badness, and emotional intensity on scales from 0 to 9 . Although analyses of bad and good ratings separately would be of interest, these ratings were highly negatively correlated (mean $r=.83$ ) and thus highly redundant. Consequently, it would not be meaningful to use both as re- gression parameters in our analyses. Given this redundancy, we calculated valence as the participant's rating for good minus the rating for bad for each concept (see Cunningham et al., 2003; Cunningham et al., 2004). Following these ratings, the participants completed the short questionnaire that measured promotion and prevention regulatory orientations. Aspects of the data from this study that did not involve individual differences in promotion/prevention focus are reported in Cunningham et al. (2004).

\section{fMRI Parameters}

All imaging was conducted with a Siemens $3 \mathrm{~T}$ scanner at the Yale Magnetic Resonance Research Center. For whole-brain functional coverage, 32 axial slices (slice thickness $=3.8 \mathrm{~mm}$, no skip) were prescribed parallel to the AC-PC line, with the 11 th slice centered on the AC-PC line. Nearly isotropic functional images were acquired from inferior to superior using a single-shot gradient echo planar pulse sequence $(\mathrm{TE}=25 \mathrm{msec}, \mathrm{TR}=2 \mathrm{sec}$, in-plane resolution $=3.75 \times 3.75 \mathrm{~mm}$, matrix size $=64 \times 64$, and FOV $=24 \times$ $24 \mathrm{~cm})$.

\section{Data Analysis}

Data were analyzed using the general linear model as implemented in SPM2 (Friston et al., 1995). Prior to analysis, data were corrected for slice acquisition time. Motion correction was performed using the INRIAlign toolbox for SPM (Freire \& Mangin, 2001). Data were then transformed to conform to the default EPI MNI brain interpolated to $4 \times 4 \times 4 \mathrm{~mm}$. Functional data were smoothed using a 12-mm FWHM (full-width-half-maximum) kernel. Finally, a low-pass filter removed frequencies greater than $0.18 \mathrm{~Hz}$, a cutoff that represents the frequency after which signals as a function of experimental effects are no longer expected.

Regression analyses were conducted to examine the relationship between the participants' ratings on attitude valence separately for the good/bad task and the abstract/concrete task. For this analysis, a series of regressors were constructed to examine brain activity for each of the trial types using the BOLD fMRI signal. Two regressors were used for each effect: the expected BOLD signal following neural activity and a time derivative. For each participant and judgment type (good/bad, abstract/concrete), covariation with these parametric regressors identified brain regions where BOLD signal changes were significantly related to concept valence. In addition, for every trial, we included a regressor for emotional intensity regardless of valence. Thus, the effects of valence represent partial correlations, with the effects of emotional intensity removed (see Cunningham et al., 2004). Contrast maps were generated for each participant for each analysis.

For each participant, valence contrast maps were generated that corresponded to increases or decreases in brain activity associated with the processing of valence (defined here as good/bad). For each map, negative voxel values corresponded to more activity for negative than for positive stimuli, and positive values corresponded to more activity for positive than for negative stimuli. Using these contrast maps as input, we regressed each participant's valence effect with both promotion and prevention scores. These parameters were entered simultaneously to allow us to examine the independent influences of promotion and prevention focus. Although promotion and prevention focus are conceptually (Higgins, 1997, 1998) and statistically (Cunningham, Farb, \& Nezlek, 2005; Farb \& Cunningham, 2005) orthogonal, we did observe a small positive correlation between prevention and promotion focus in this sample $(r=.21)$. It should be noted that the observed correlation was positive, not negative, indicating that prevention and promotion focus are not simply reciprocals of one another. Areas that correlate positively with promotion (or prevention) focus reflect regions in which greater focus corresponds to greater activity for positive stimuli, and areas that correlate negatively reflect regions that are associated with greater activity for negative stimuli. 
Table 1

Good/Bad Task: Areas Showing Association Between Regulatory Orientation and Valence

\begin{tabular}{|c|c|c|c|c|c|c|}
\hline \multicolumn{7}{|c|}{ Regulatory orientation and valence } \\
\hline Size & Area & BA & $t$ & $x$ & $y$ & $z$ \\
\hline \multicolumn{7}{|c|}{ Negative Valence (Good/Bad) Correlation With Prevention } \\
\hline 31 & Amygdala & $\mathbf{L}$ & 5.26 & -20 & -8 & -8 \\
\hline 35 & Amygdala & $\mathbf{R}$ & 4.81 & 20 & -8 & -8 \\
\hline \multirow[t]{2}{*}{54} & Anterior cingulate & 32 & 4.55 & -4 & 36 & 16 \\
\hline & Lateral orbital gyrus & $\mathrm{R} 38 / 47$ & 4.14 & 40 & 20 & -16 \\
\hline 64 & Extrastriate cortex & 18 & 4.94 & 4 & -84 & 16 \\
\hline 102 & Precentral gyrus & R6 & 7.29 & 48 & -8 & 52 \\
\hline 27 & Heschl's gyrus & $\mathrm{R} 48$ & 5.56 & 52 & -8 & 8 \\
\hline 31 & Superior occipital gyrus & L18 & 5.48 & -12 & -96 & 28 \\
\hline \multirow[t]{2}{*}{27} & Middle occipital gyrus & L17 & 4.79 & -16 & -100 & 8 \\
\hline & Calcarine gyrus & L17 & 4.55 & -12 & -100 & 0 \\
\hline \multirow[t]{2}{*}{231} & Temporal pole/lateral orbital gyrus & $\mathrm{L} 38 / 47 / 48$ & 5.90 & -44 & 4 & -12 \\
\hline & Pallidum & $\mathrm{L}$ & 5.26 & -20 & -8 & -8 \\
\hline \multirow[t]{2}{*}{62} & Pallidum & $\mathrm{R}$ & 5.98 & 24 & -12 & -4 \\
\hline & Putamen & $\mathrm{R}$ & 5.35 & 36 & -12 & -4 \\
\hline \multirow[t]{2}{*}{254} & Postcentral gyrus & L3 & 6.33 & -24 & -36 & 60 \\
\hline & Middle cingulum & 24 & 5.37 & -8 & -4 & 40 \\
\hline \multicolumn{7}{|c|}{ Positive Valence (Good/Bad) Correlation With Promotion } \\
\hline 7 & Amygdala & $\mathbf{L}$ & 2.75 & -16 & -4 & -12 \\
\hline * & Amygdala & $\mathbf{R}$ & 2.37 & 16 & -4 & -24 \\
\hline 102 & Anterior cingulate & 32 & 5.21 & -4 & 44 & 16 \\
\hline 117 & Extrastriate cortex & 18 & 6.83 & 8 & -88 & 12 \\
\hline 28 & Precentral gyrus & R6 & 6.53 & 52 & -8 & 56 \\
\hline \multirow[t]{2}{*}{49} & Extrastriate cortex & $\mathrm{R} 17$ & 4.54 & 12 & -60 & 12 \\
\hline & Precuneus & 29 & 4.45 & -8 & -48 & 12 \\
\hline 15 & Precuneus & 23 & 4.18 & -4 & -56 & 36 \\
\hline 15 & Temporal pole & $\mathrm{R} 38$ & 4.38 & 28 & 20 & -28 \\
\hline
\end{tabular}

Note-Table shows local maxima ( $p<.001 ; p<.01$ for amygdala) with an extent threshold of 15 voxels. ${ }^{*}$ The correlation of right amygdala and promotion is significant at $p<.05$ (see text). BA, Brodmann's area; $\mathrm{R} / \mathrm{L}$, right or left hemisphere; $t$, maximal $t$ statistic for the statistical difference; $x, y, z, 3$-D coordinates of activation within normalized MNI space. Areas discussed in the text are in bold.

To identify significant regions of activation at the group level, random-effects composite group $t$ maps were generated using the individual participant contrast maps as input. Regions of activation were defined as those areas in which 15 contiguous voxels were significant at $p<.001$. This was done for all regions except for the amygdala, an a priori region of interest based on previous published work and data from our own lab. For the amygdala, region of interest analyses were performed using an anatomical mask $(p<.01$, with a cluster value of 5 ) that provided a correction for Type I errors.

\section{RESULTS}

\section{Interaction of Valence and Regulatory Focus: Good/Bad Task}

We first report areas that were negatively correlated with prevention orientation and positively correlated with promotion orientation (see Table 1). These regions show a fit between regulatory orientation and stimulus valence - that is, associations between prevention orientation and stimulus negativity and between promotion orientation and stimulus positivity. Four regions show this pattern: the amygdala, anterior cingulate, extrastriate cortex, and right precentral gyrus.

Amygdala activation varied as a function of regulatory focus, so that greater activation to negative stimuli was associated with prevention focus [left, $t(19)=4.81, p<$
.001 ; right, $t(19)=5.26, p<.001]$ and greater activation to positive stimuli was associated with promotion focus [left, $t(19)=2.75, p<.01]$ (see Figure 1$)$. When the threshold was reduced to $p<.05$, a smaller region of right amygdala also showed greater activation to positive stimuli associated with promotion focus $[t(19)=2.37]$. These findings provide additional evidence relevant to a long-standing debate regarding whether amygdala activation represents the processing of negativity or of emotional intensity. As noted earlier, although the preponderance of studies have found greater amygdala activation with negative than with positive stimuli, activation has also been found with positive as compared with neutral stimuli. Our finding that amygdala activation changes as a function of regulatory focus provides further converging evidence that the amygdala signals important (e.g., motivationally relevant) stimuli in the environment. These stimuli are often negative- - but for particular motivational states, they can be positive.

Anterior cingulate cortex (ACC) has been associated with control (Cohen, Botvinick, \& Carter, 2000), attentional (Kondo, Osaka, \& Osaka, 2004), and emotional (Matthews, Paulus, Simmons, Nelesen, \& Dimsdale, 2004; Taylor, Phan, Decker, \& Liberzon, 2003) processes. The ACC has been associated with attentional 


\section{Prevention Focus, Negative Correlation}
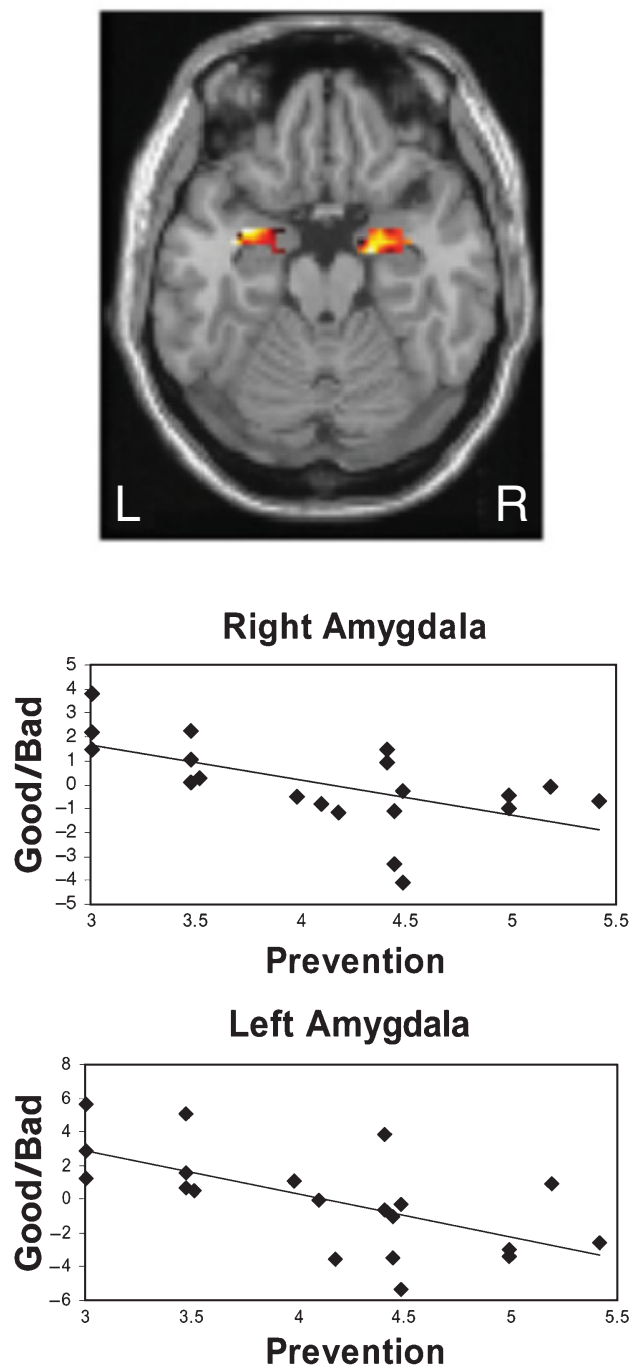
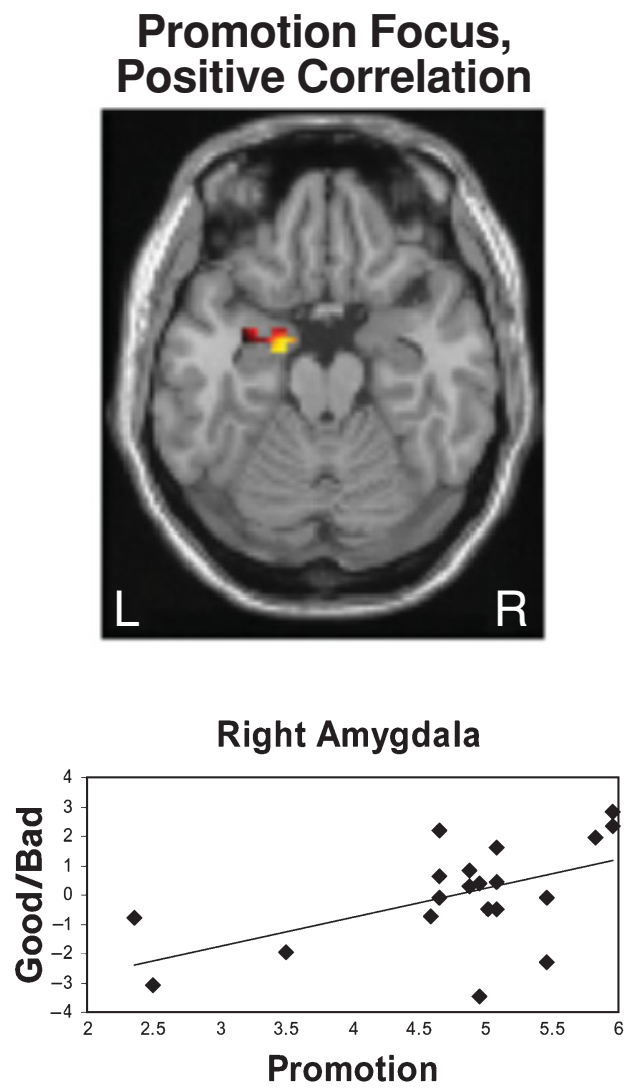

Figure 1. Areas of amygdala showing an association between regulatory focus and valence. Each axial image $(z=-20)$ reflects regression parameters in which activity to valenced stimuli (good/bad) was associated with individual differences in prevention and promotion focus. Parameters are partial correlations between valence (good/bad) and prevention after controlling for promotion or the converse. Scatterplots reflect these partial correlations. Note that all relationships remain significant even when the 3 participants with the lowest promotion scores are dropped.

processes that select among stimuli for action (Posner, Petersen, Fox, \& Raichle, 1988) or thought (Johnson et al., in press, Experiment 5). Individuals with a prevention or promotion focus can be characterized as tending to select different stimuli for action: positive stimuli (promotion focus) or negative stimuli (prevention focus). In agreement with this pattern, we found that activity in ACC interacted with valence and regulatory focus in the same way as activity in the amygdala, with greater sensitivity to negative stimuli for prevention focus $[t(19)=$ $4.55, p<.001]$ and to positive stimuli for promotion focus $[t(19)=5.21, p<.001]($ see Figure 2$)$.
If positive and negative information differentially capture attention as a function of regulatory focus, then regulatory focus may be associated not only with activity in ACC, but also with activity in perceptual areas. In agreement with this hypothesis, we found that activation in the extrastriate cortex interacted with valence and regulatory focus so that increasing sensitivity to negative stimuli was associated with prevention focus $[t(19)=4.94, p<$ $.001]$ and increasing sensitivity to positive stimuli was associated with promotion focus $[t(19)=6.83, p<$ $.001]$ (see Figure 2). Because the same stimuli are processed differently according to regulatory orientation, 


\section{Prevention Focus, Negative Correlation}
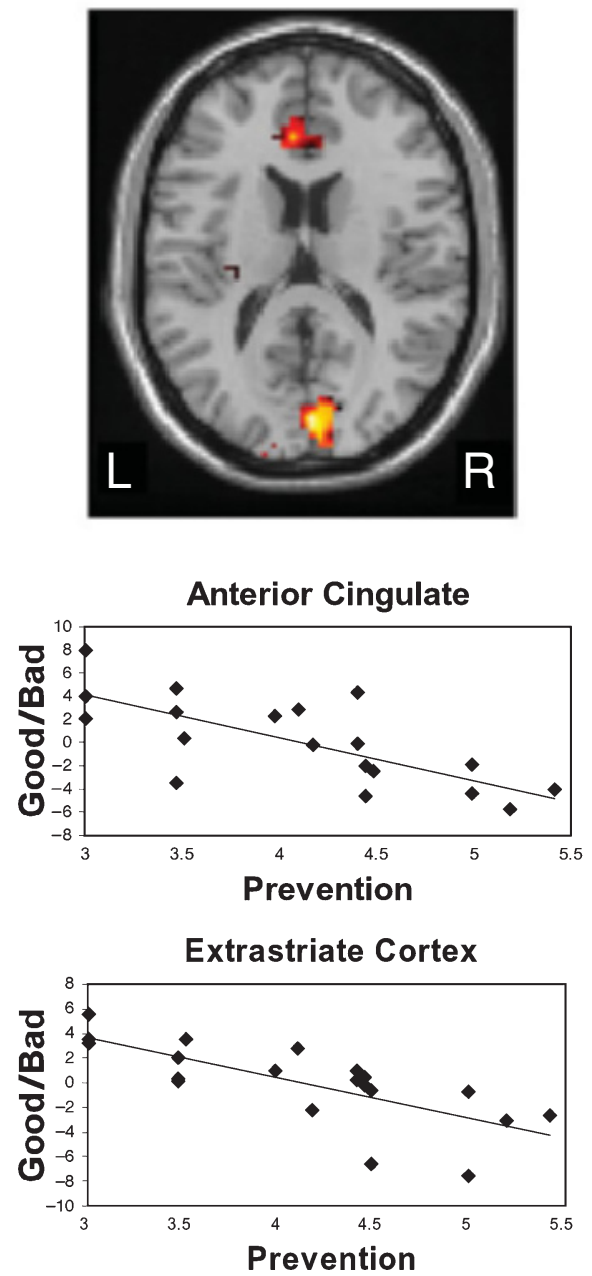

\section{Promotion Focus, Positive Correlation}

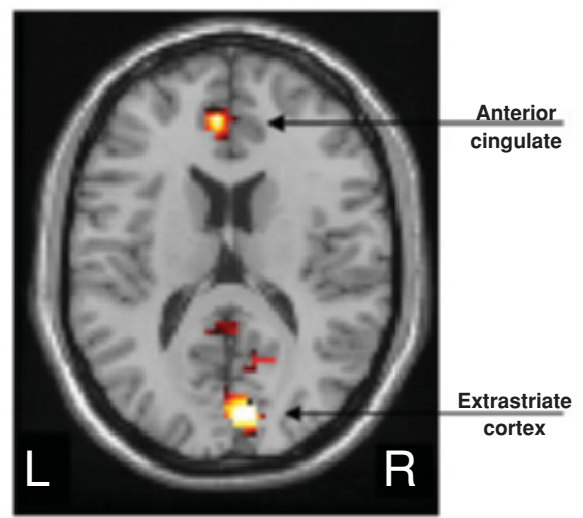

Anterior Cingulate
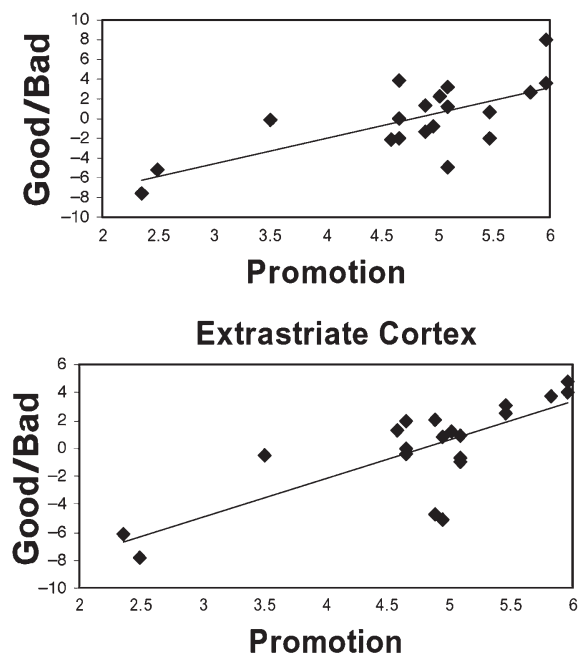

Figure 2. Areas of anterior cingulate and extrastriate cortex showing an association between regulatory focus and valence. Each axial image $(z=16)$ reflects regression parameters in which activity to valenced stimuli (good/bad) was associated with individual differences in prevention and promotion focus. Parameters are partial correlations, so results reflect an association with prevention after promotion or the converse is controlled for. Scatterplots reflect partial correlations between prevention/promotion and significant voxels. Note that all relationships remain significant even when the 3 participants with the lowest promotion scores are dropped.

this finding cannot be explained as being a function of perceptual features of the stimuli. Motivationally relevant stimuli may be processed more quickly and for longer periods of time.

Finally, an area of right precentral gyrus was found that mirrored the patterns found for the amygdala, anterior cingulate, and extrastriate cortex [prevention, $t(19)=7.29, p<.001$; promotion, $t(19)=6.53, p<$ $.001]$. Although the functional role of the right precentral gyrus is less clear than that of other regions, activation in this region has been found for feeling states associated with emotional experience-for instance, with self-reported experienced emotion (Canli, Desmond,
Zhao, \& Gabrieli, 2002), induced emotional feelings (Teasdale et al., 1999), or the feeling of familiarity when making preference judgments (Elliott \& Dolan, 1998). Future research will need to further examine the role of this area in the processes of evaluation.

\section{Specific Interactions With Promotion and Prevention Focus}

In addition to regions that responded in opposite ways for promotion and prevention orientations, we found several other regions that only correlated negatively with prevention focus (Table 1). These areas included additional sensory areas, an additional limbic area associated 
with emotion (temporal pole), and lateral orbital cortex, which has been associated with emotional processing (Anderson et al., 2003; Cunningham et al., 2004; Rule, Shimamura, \& Knight, 2002; Shimamura, 2000). Interestingly, for the good/bad task, no regions were identified as positive correlations with prevention focus or negative correlations with promotion focus (see Table 1).

\section{Interaction of Valence and Regulatory Focus: Abstract/Concrete Task}

Whereas regions associated systematically with regulatory focus were found for the good/bad task, no such regions were found for the abstract/concrete task. This finding suggests that the effects of regulatory focus on attitudes may be substantially more pronounced for explicit than for implicit evaluative judgments. To test this interaction, nested LISREL models were compared in order to determine whether the regression paths for the good/bad task were significantly stronger than for the abstract/concrete task for each of the reported regions (e.g., bilateral amygdala, anterior cingulate, extrastriate cortex, and right precentral gyrus). In the first of two models, separate regression paths were estimated for the good/bad task and the abstract/concrete task. For the second model, the regression paths for the two tasks were constrained to have the same value. For every region, the constrained model introduced a significant degree of error. This pattern of results indicates that the regression paths were significantly stronger in the good/bad task than in the abstract/concrete task for both the prevention regression weights [right amygdala, $\chi^{2}(1)=12.66, p<.001$; left amygdala, $\chi^{2}(1)=6.98$, $p<.01$; anterior cingulate, $\chi^{2}(1)=16.15, p<.001$; extrastriate cortex, $\chi^{2}(1)=11.06, p<.001$; right precentral gyrus, $\left.\chi^{2}(1)=13.56, p<.001\right]$ and the promotion regression weights [right amygdala, $\chi^{2}(1)=5.48, p<$ .05 ; left amygdala, $\chi^{2}(1)=3.83, p=.051$; anterior cingulate, $\chi^{2}(1)=17.50, p<.001$; extrastriate cortex, $\chi^{2}(1)=7.17, p<.01$; right precentral gyrus, $\chi^{2}(1)=$ $13.83, p<.001]$. A list of regions in which either promotion or prevention orientation interacted with stimulus valence in the abstract/concrete task is reported in Table 2.

Table 2

Abstract/Concrete Task: Areas Showing Association Between Regulatory Orientation and Valence

\begin{tabular}{|c|c|c|c|c|c|c|}
\hline Size & Area & BA & $t$ & $x$ & $y$ & $z$ \\
\hline \multicolumn{7}{|c|}{ Positive Valence (Good/Bad) Correlation With Prevention } \\
\hline 25 & Middle occipital gyrus & L19 & 5.32 & -44 & -84 & 28 \\
\hline \multicolumn{7}{|c|}{ Negative Valence (Good/Bad) Correlation With Promotion } \\
\hline 19 & Middle frontal gyrus & R9 & 5.69 & 48 & 12 & 52 \\
\hline 20 & Superior frontal gyrus & R8 & 4.83 & 12 & 24 & 60 \\
\hline 48 & Medial frontal gyrus & 9 & 4.80 & 8 & 36 & 40 \\
\hline
\end{tabular}

Note-The table shows local maxima $(p<.001)$ with an extent threshold of 15 voxels. BA, Brodmann's area; R/L, right or left hemisphere; $t$, maximal $t$ statistic for the statistical difference; $x, y, z, 3-\mathrm{D}$ coordinates of activation within normalized MNI space.

\section{DISCUSSION}

During an evaluative task in which participants made judgments about the valence of concepts, we found activation in a common set of brain regions for stimuli that fit with an individual's self-regulatory focus. The areas involved in this system included limbic (amygdala and cingulate cortex) and perceptual (extrastriate cortex) areas. For each of these regions, greater sensitivity to positive stimuli was associated with a promotion focus, and greater sensitivity to negative stimuli was associated with a prevention focus. Together, these areas suggest a system for vigilance, and selection for action, that is tuned to detect motivationally significant stimuli. The present study was designed to investigate individual differences in regulatory focus that are, presumably, relatively stable over time. However, within individuals regulatory focus should also reflect situational specificity (Higgins, 1997, 1998); thus, in the future, neuroimaging studies manipulating focus would be of interest.

These results identify neural correlates for the important distinction in motivational focus identified by Higgins $(1997,1998)$. At the same time, our results do not suggest that two different self-regulatory motivational systems underlie differences in the aspects of evaluative information to which people are sensitive. Although there may be different approach and avoidance systems (Sutton \& Davidson, 1997), our results suggest that selfregulatory focus may involve a common system that underlies a bias in attention and vigilance toward positive or negative stimuli. However, we found brain regions (e.g., temporal pole and lateral orbital cortex) that correlated negatively with prevention focus but did not correlate with promotion focus. Thus, whether promotion and prevention are associated with a common neural system or two different systems depends on whether one emphasizes common or different regions. Additional work is needed to clarify how regulatory system(s) or subsystem(s) should be defined at the neural level.

One finding that is particularly striking from these data is that we found a near perfect symmetry of brain regions sensitive to valenced information - with a positive relationship for one motivational orientation and a negative relationship for another. This result contrasts with those from previous work on introversion/neuroticism and BIS/BAS, which has found different sets of regions for each of the contrasting individual-difference measures. This suggests that goal or motivational state may be a function of a single set of brain regions that may then recruit additional processes, such as those that direct approach or avoidance behavior.

Previous research has suggested that the amygdala responds to stimuli that have high subjective incentive value (Arana et al., 2003). This finding, in combination with the present results showing differential amygdala activation to positive and negative stimuli as a function of self-regulatory focus, is consistent with hypotheses 
that the amygdala is involved in vigilance (see Davis \& Whalen, 2001; Whalen, 1998), unconsciously responding to perceptual information from the environment about stimuli relevant for information processing goals. Although the effects of "bad" stimuli may be almost universally more powerful than those of "good" stimuli (Baumeister, Bratslavsky, Finkenauer, \& Vohs, 2001), and thus "bad" stimuli are the ones that primarily capture attention and are more arousing, for some processing goals positive stimuli may acquire these properties. The present results regarding valence and the amygdala are particularly striking, because the potentially confounding influence of emotional arousal was partialed out from all analyses. Our data add to the growing consensus that amygdala activation may be more closely associated with the processing of "important" stimuli rather than only of negative (e.g., fearful) stimuli.

This idea is consistent with a suggestion of Whalen (1998), who proposed that amygdala processing is greatest for stimuli that are ambiguous or when more information must be extracted. Although one could make the argument that stimuli that fit with one's regulatory focus should be processed more efficiently, it is also possible that more subtle distinctions are made among stimuli that are more relevant to regulatory focus, and thus that such stimuli require more processing. Within this context, people with different regulatory foci are vigilant toward different stimuli and for different reasons. Once detected, a common network may direct attention toward these motivationally relevant stimuli for additional processing.

The idea that stimuli that fit a self-regulatory focus receive greater attention/cognitive processing is supported by differences in correlations in the anterior cingulate and extrastriate cortex. Within this context, missing a potentially positive stimulus is more important in promotion focus, and missing a potentially negative stimulus is more important in prevention focus. Our data suggest that the anterior cingulate may play an important role, together with the amygdala, in this system to identify and potentially direct attention to motivationally relevant stimuli as a function of regulatory focus. Such an interpretation is consistent with the proposed role of more rostral areas of anterior cingulate cortex. Specifically, these particular areas of anterior cingulate may be involved in the motivational component of an attentional network. These areas show more activity in many tasks that require interactions between emotion and attention, such as emotional Stroop tasks (Bush et al., 1998), emotional oddball tasks (Fichtenholtz et al., 2004), and emotional spatial attention tasks (Vuilleumier, Armony, Driver, \& Dolan, 2001).

Moreover, we found that regulatory focus was related to activity in the extrastriate cortex, so that greater activation was found to stimuli that fit an individual's selfregulatory focus. Given that the same stimuli were processed differently for different people, this finding cannot be attributed to differences in the perceptual com- plexity of the stimuli and likely reflects greater attention to motivationally relevant stimuli. Previous findings have observed activation of visual cortex to either emotionally evocative or negative stimuli (Bradley et al., 2003). Our findings suggest, especially since significant correlations between regulatory focus and brain activity were only observed in the good/bad task, that the capture of attention by arousing stimuli may reflect a top-down effect on perceptual areas driven by motivation rather than reflecting perceptual aspects of the stimuli alone.

In summary, this article shows that individual differences in self-regulatory focus are associated with neural differences in the way that valenced (good and bad) information is processed. Interestingly, a common set of brain regions involved in emotional arousal and attentional processing appears to be tuned to either positive or negative information for promotion or prevention focus, respectively. Thus, motivation plays an important role in determining which aspects of stimuli attentional networks will be sensitive to and vigilant toward. These findings highlight the value of investigating neural data in light of individual-difference variables. By studying individual differences in evaluative judgments, both our understanding of the social cognitive processes of evaluation and the brain processes that give rise to them may be clarified.

\section{REFERENCES}

Adolphs, R., \& Tranel, D. (2004). Impaired judgments of sadness but not happiness following bilateral amygdala damage. Journal of Cognitive Neuroscience, 16, 453-462.

Adolphs, R., Tranel, D., Hamann, S., Young, A. W., Calder, A. J., Phelps, E. A., Anderson, A., Lee, G. P., \& Damasio, A. R. (1999). Recognition of facial emotion in nine individuals with bilateral amygdala damage. Neuropsychologia, 37, 1111-1117.

Anderson, A. K., Christoff, K., Stappen, I., Panitz, D., Ghahremani, D. G., Glover, G., Gabrieli, J. D. E., \& Sobel, N. (2003). Dissociated neural representations of intensity and valence in human olfaction. Nature Neuroscience, 6, 196-202.

Anderson, A. K., \& Phelps, E. A. (2001). Lesions of the human amygdala impair enhanced perception of emotionally salient events. $\mathrm{Na}$ ture, 411, 305-309.

Anderson, A. K., Spencer, D. D., Fulbright, R. K., \& Phelps, E. A. (2000). Contribution of the anteromedial temporal lobes to the evaluation of facial emotion. Neuropsychology, 14, 526-536.

Arana, F. S., Parkinson, J. A., Hinton, E., Holland, A. J., Owen, A. M., \& Roberts, A. C. (2003). Dissociable contributions of the human amygdala and orbitofrontal cortex to incentive motivation and goal selection. Journal of Neuroscience, 23, 9632-9638.

Baumeister, R. F., Bratslavsky, E., Finkenauer, C., \& Vohs, K. D. (2001). Bad is stronger than good. Review of General Psychology, 5, 323-370.

Bradley, M. M., Sabatinelli, D., Lang, P. J., Fitzsimmons, J. R., KING, W., \& DesaI, P. (2003). Activation of the visual cortex in motivated attention. Behavioral Neuroscience, 117, 369-380.

Bush, G., Whalen, P. J., Rosen, B. R., Jenike, M. A., McInerney, S. C., \& RaUch, S. L. (1998). The counting Stroop: An interference task specialized for functional neuroimaging - Validation study with functional MRI. Human Brain Mapping, 6, 270-282.

Canli, T., Desmond, J. E., Zhao, Z., \& Gabrieli, J. D. E. (2002). Sex differences in the neural basis of emotional memories. Proceedings of the National Academy of Sciences, 99, 10789-10794. 
Canli, T., Sivers, H., Whitfield, S. L., Gotlib, I. H., \& Gabrieli, J. D. E. (2002). Amygdala response to happy faces as a function of extraversion. Science, 296, 2191.

Canli, T., Zhao, Z., Desmond, J. E., Kang, E., Gross, J., \& Gabrieli, J. D. E. (2001). An fMRI study of personality influences on brain reactivity to emotional stimuli. Behavioral Neuroscience, 115, 33-42.

Cesario, J., Grant, H., \& Higgins, E. T. (2004). Regulatory fit and persuasion: Transfer from "feeling right." Journal of Personality \& Social Psychology, 86, 388-404.

Cohen, J. D., Botvinick, M., \& Carter, C. S. (2000). Anterior cingulate and prefrontal cortex: Who's in control? Nature Neuroscience, $\mathbf{3}$, 421-423.

Cunningham, W. A., Farb, N., \& Nezlek, J. B. (2005). [Prevention and promotion: A personality analysis]. Unpublished raw data, University of Toronto.

Cunningham, W. A, Johnson, M. K., Gatenby, J. C., Gore, J. C., \& BANAJI, M. R. (2003). Component processes of social evaluation. Journal of Personality \& Social Psychology, 85, 639-649.

Cunningham, W. A., Raye, C. L., \& Johnson, M. K. (2004). Implicit and explicit evaluation: fMRI correlates of valence, emotional intensity, and control in the processing of attitudes. Journal of Cognitive Neuroscience, 16, 1717-1729.

Davis, M., \& Whalen, P. J. (2001). The amygdala: Vigilance and emotion. Molecular Psychiatry, 6, 13-34.

Elliott, R., \& Dolan, R. J. (1998). Neural response during preference and memory judgments for subliminally presented stimuli: A functional neuroimaging study. Journal of Neuroscience, 18, 4697-4704.

FARB, N., \& CUNNINGHAM, W. A. (2005). [A multitrait multimethod analysis of prevention and promotion]. Unpublished raw data, University of Toronto.

Fichtenholtz, H. M., Dean, H. L., Dillon, D. G., Yamasaki, H., McCARTHY, G., \& LABAR, K. S. (2004). Emotion-attention network interactions during a visual oddball task. Cognitive Brain Research, 20, 67-80.

FreIRE, L., \& MANGIN, J. F. (2001). Motion correction algorithms may create spurious brain activations in the absence of subject motion. NeuroImage, 14, 709-722.

Freitas, A. L., \& Higgins, E. T. (2002). Enjoying goal-directed action: The role of regulatory fit. Psychological Science, 13, 1-6.

Friston, K. J., Holmes, A. P., Worsley, K. J., Poline, J. P., Frith, C. D., \& FRACKOWIAK, R. S. J. (1995). Statistic parametric maps in functional imaging: A general linear approach. Human Brain Mapping, 2, 189-210.

Garavan, H., Pendergrass, J. C., Ross, T. J., Stein, E. A., \& Risinger, R. C. (2001). Amygdala response to both positively and negatively valenced stimuli. NeuroReport, 12, 2779-2783.

GraY, J. A. (1981). A critique of Eysenck's theory of personality. In H. J. Eysenck (Ed.), A model for personality (pp. 246-276). New York: Springer-Verlag.

Gray, J. A. (1982). The neuropsychology of anxiety. New York: Oxford University Press.

Hamann, S. B., Ely, T. D., Hoffman, J. M., \& Kilts, C. D. (2002). Ecstasy and agony: Activation of the human amygdala in positive and negative emotion. Psychological Science, 13, 135-141.

HamanN, S. [B.], \& MaO, H. (2002). Positive and negative emotional verbal stimuli elicit activity in the left amygdala. NeuroReport, 13, 15-19.

Higgins, E. T. (1997). Beyond pleasure and pain. American Psychologist, 52, 1280-1300.

HigGins, E. T. (1998). Promotion and prevention: Regulatory focus as a motivational principle. Advances in Experimental Social Psychology, 46, 1-46.

Higgins, E. T. (2000). Making a good decision: Value from fit. American Psychologist, 55, 1217-1230.

Higgins, E. T., Idson, L. C., Freitas, A. L., Spiegel, S., \& Molden, D. C. (2003). Transfer of value from fit. Journal of Personality \& Social Psychology, 84, 1140-1153.

Idson, L. C., Liberman, N., \& Higgins, E. T. (2000). Distinguishing gains from nonlosses and losses from nongains: A regulatory focus perspective on hedonic intensity. Journal of Experimental Social Psychology, 36, 252-274.
Isenberg, N., Silbersweig, D., Engelien, A., Emmerich, S. Malavade, K., Beattie, B., Leon, A. C., \& Stern, E. (1999). Linguistic threat activates the human amygdala. Proceedings of the $\mathrm{Na}$ tional Academy of Sciences, 96, 10456-10459.

Johnson, M. K., Raye, C. L., Mitchell, K. J., Greene, E. J., CunNINGHAM, W. A., \& SANISLOW, C. A. (in press). Using fMRI to investigate a component process of reflection: Prefrontal correlates of refreshing a just-activated representation. Cognitive, Affective, \& Behavioral Neuroscience.

Kondo, H., OsaKa, N., \& OsaKa, M. (2004). Cooperation of the anterior cingulate cortex and dorsolateral prefrontal cortex for attention shifting. NeuroImage, 23, 670-679.

LaBar, K. S., Gatenby, J. C., Gore, J. C., LeDoux, J. E., \& Phelps, E. A. (1998). Human amygdala activation during conditioned fear acquisition and extinction: A mixed-trial fMRI study. Neuron, 20, 937-945.

LaBar, K. S., LeDoux, J. E., Spencer, D. D., \& Phelps, E. A. (1995). Impaired fear conditioning following unilateral temporal lobectomy in humans. Journal of Neuroscience, 15, 6846-6855.

LeDoux, J. E. (2000). Emotion circuits in the brain. Annual Review of Neuroscience, 23, 155-184.

LeE, A. Y., \& AAKER, J. L. (2004). Bringing the frame into focus: The influence of regulatory fit on processing fluency and persuasion. Journal of Personality \& Social Psychology, 86, 205-218.

Liberzon, I., Phan, K. L., Decker, L. R., \& TaYlor, S. F. (2003). Extended amygdala and emotional salience: A PET activation study of positive and negative affect. Neuropsychopharmacology, 28, 726733.

Matthews, S. C., Paulus, M. P., Simmons, A. N., Nelesen, R. A., \& Dimsdale, J. E. (2004). Functional subdivisions within anterior cingulate cortex and their relationship to autonomic nervous system function. Neurolmage, 22, 1151-1156.

Morris, J. S., Frith, C. D., Perrett, D. I., Rowland, D., Young, A. W., CAlder, A. J., \& Dolan, R. J. (1996). A differential neural response in the human amygdala to fearful and happy facial expressions. $\mathrm{Na}$ ture, 383, 812-815.

Pervin, L. A. (1994). A critical analysis of current trait theory. Psychological Inquiry, 5, 103-113.

Phelps, E. A., O'Connor, K. J., Gatenby, J. C., Gore, J. C., GrilLON, C., \& DAVIS, M. (2001). Activation of the left amygdala to a cognitive representation of fear. Nature Neuroscience, 4, 437-441.

Posner, M. I., Petersen, S. E., Fox, P. T., \& Raichle, M. E. (1988). Localization of cognitive operations in the human brain. Science, 240, 1627-1631.

Reuter, M., Stark, R., Hennig, J., Walter, B., Kirsch, P. Schienle, A., \& Vaitl, D. (2004). Personality and emotion: Test of Gray's personality theory by means of an fMRI study. Behavioral Neuroscience, 118, 462-469.

Rule, R. R., Shimamura, A. P., \& Knight, R. T. (2002). Orbitofrontal cortex and dynamic filtering of emotional stimuli. Cognitive, Affective, \& Behavioral Neuroscience, 2, 264-270.

Shimamura, A. P. (2000). The role of the prefrontal cortex in dynamic filtering. Psychobiology, 28, 207-218.

Spiegel, S., Grant-Pillow, H., \& Higgins, E. T. (2004). How regulatory fit enhances motivational strength during goal pursuit. European Journal of Social Psychology, 34, 39-54.

SutTon, S. K., \& Davidson, R. J. (1997). Prefrontal brain asymmetry: A biological substrate of the behavioral approach and inhibition systems. Psychological Science, 8, 204-210.

Taylor, S. F., Phan, K. L., Decker, L. R., \& Liberzon, I. (2003). Subjective rating of emotionally salient stimuli modulates neural activity. NeuroImage, 18, 650-659.

Teasdale, J. D., Howard, R. J., Cox, S. G., Ha, Y., Brammer, M. J., Williams, S. C. R., \& Checkley, S. A. (1999). Functional MRI study of the cognitive generation of affect. American Journal of Psychiatry, 156, 209-215.

Vuilleumier, P., Armony, J. L., Driver, J., \& Dolan, R. J. (2001). Effects of attention and emotion on face processing in the human brain: An event-related fMRI study. Neuron, 30, 829-841.

Whalen, P. J. (1998). Fear, vigilance, and ambiguity: Initial neuroimaging studies of the human amygdala. Current Directions in Psychological Science, 7, 177-188. 
Zald, D. H., Lee, J. T., Fluegel, K. W., \& Pardo, J. V. (1998). Aversive gustatory stimulation activates limbic circuits in humans. Brain, 121, 1143-1154.

ZALD, D. H., \& PARDo, J. V. (1997). Emotion, olfaction, and the human amygdala: Amygdala activation during aversive olfactory stimulation. Proceedings of the National Academy of Sciences, 94, 41194124 .

\section{NOTE}

1. In terms of potential relations among these various individualdifference constructs, Cunningham, Farb, and Nezlek (2005) found that more promotion-focused individuals tend to be slightly more extroverted and open to experience than less promotion-focused individuals. In addition, prevention-focused individuals tend to be slightly more neurotic.

(Manuscript received October 15, 2004;

revision accepted for publication April 18, 2005.) 